\title{
Direkt gewählte Ministerpräsidenten der Länder - eine kritische Folgenabschätzung der von Frank Decker in Heft 3/2010 der ZParl veröffentlichten Überlegungen
}

\author{
Everhard Holtmann
}

In Heft 3/2010 der ZParl stellt Frank Decker seine Überlegungen vor, wie jene plebiszitären Verfahren, die in die Landesverfassungen der deutschen Bundesländer bereits Eingang gefunden haben (Volksinitiativen, Volksbegehren, Volksentscheide), mit dem - Bund wie Länder gleichermaßen kennzeichnenden - Basismodell des repräsentativen Regierungssystems konstitutionell besser ausbalanciert werden könnten. ${ }^{1}$ Ausgehend von der Annahme, dass die volksunmittelbare Gesetzesinitiative „Vereinbarkeitsprobleme mit dem herkömmlichen parlamentarischen Entscheidungsverfahren“ aufwerfe, plädiert Decker mit Blick auf die Landesebene für den Wechsel vom parlamentarischen zum präsidentiellen System. Mit Einführung der Direktwahl der Ministerpräsidenten würden, so Decker, die Länder einen auf kommunaler Ebene seit den 1990er Jahren bereits erfolgten Reformschritt nachvollziehen, was im übrigen sachpolitisch durch den ähnlichen Aufgabenzuschnitt beider Ebenen begründbar sei. Ein solcher „Wechsel zum präsidentiellen Modell“ habe „unter Demokratiegesichtspunkten gleich einen doppelten Vorteil: Einerseits würde man so die bundespolitische Überlagerung der Landtagswahlen zurückdrehen und der Landespolitik wieder ein stärkeres eigenständiges Gewicht einräumen, andererseits wäre - gleichsam als Nebeneffekt - eine bessere Integration der Volksgesetzgebung möglich“2.

Gegenüber dieser These sei zunächst ein kategorialer Vorbehalt geäußert. Die Überlegung, ein „Wechsel zum präsidentiellen Modell“ ermögliche eine höhere Kompatibilität mit landesverfassungsrechtlichen Instrumenten der Volksgesetzgebung, gründet offenbar auf der Prämisse, es handele sich um zwei Verfahrensvarianten unter dem einen Oberbegriff Direkte Demokratie. Diese Gleichsetzung von Volkswahl und Volksgesetzgebung leistet einer begrifflich-systematischen Unschärfe Vorschub. Zudem ist der Nachweis, dass die verfassungsförmige Koexistenz beider Komponenten ein Regierungssystem in sich passfähiger im Sinne gesteigerter Leistungskraft macht, meines Wissens bisher nicht erbracht.

Nun zu den inhaltlichen Fragen, die mit dem Präsidentialisierungsvorschlag aufgeworfen werden. Tatsächlich stellt sich das wahre Ausmaß der Überformung der Landtagswahlen durch Einflüsse der Bundespolitik, neueren ländervergleichenden Analysen zufolge, weniger dramatisch dar als zuweilen angenommen, und es ist vor allem auch von Land zu Land unterschiedlich. ${ }^{3}$ Auch erschließt sich nicht auf den ersten Blick, weshalb das Gewicht der Landespolitik infolge einer Direktwahl des Ministerpräsidenten gestärkt würde,

1 Siehe Frank Decker, Zwischen Placebo und Erfolgsmodell. Direkte Demokratie auf der Landesebene, in: ZParl, 41. Jg. (2010), H. 3, S. $564-579$.

2 Ebenda, S. 571, S. 576, in der Reihenfolge der Zitate.

3 Vgl. Oscar W. Gabriel / Everhard Holtmann, Ober sticht Unter? Zum Einfluss der Bundespolitik auf Landtagswahlen: Kontext, theoretischer Rahmen und Analysemodelle, in: ZParl; 38. Jg. (2007), H. 3, S. 445 - 462; ferner Kerstin Völkl / Kai-Uwe Schnapp / Everhard Holtmann / Oscar W. Gabriel, Wähler und Landtagswahlen in der Bundesrepublik Deutschland, Baden-Baden 2008. 
denn das im deutschen kooperativen Föderalismus bestehende Strukturmuster einer vertikal-funktionellen Gewaltenteilung von Bund und Ländern, das zutreffend als „Exekutivföderalismus" beschrieben wird, dürfte in seiner Regierungslastigkeit eher noch gefestigt werden.

Schließlich und vor allem bedürfte die demokratietheoretische Grundannahme, die auch dem Verfassungsreformvorschlag Deckers zugrunde liegt, es sei a priori wünschenswert, die bereits bestehenden plebiszitären Instrumente mittels Einfügung der Volkswahl des Ministerpräsidenten verfassungsrechtlich zu flankieren und aufzuwerten, einer kritischen Diskussion, die breiter und systematischer ansetzt und über die von Decker in besagtem Beitrag selbst gegebenen Hinweise hinausgeht. Zum oftmals gehörten Argument, direkte Demokratie sei bürgernäher als ihre parteienstaatlich mediatisierte Erscheinungsform, ist folgendes anzumerken: Nimmt man die Befunde der nationalen und ländervergleichenden empirischen Plebiszitforschung in den Blick, fällt die Bilanz aus nationaler wie internationaler Sicht insgesamt zwar nicht eindeutig aus: Volksgesetzgebung kann, wie beispielsweise eine Fallstudie zur Abstimmung über Gentechnik in der Schweiz zeigt, die deliberativen Elemente des öffentlichen Diskurses durchaus in die Richtung lenken, dass eine abstimmende Mehrheit „im Zweifel dem wirtschaftlichen Nutzen und dem technologischen Fortschritt unter den Bedingungen von Regulierung und Kontrolle den Vorzug vor dem Verzicht gibt $^{\text {“4 }}$. Andererseits sind, wie deutsche Fallstudien mehrfach belegen, in der plebiszitären Praxis die Chancen der Interessenberücksichtigung ungleich verteilt, weil gerade bei kommunalen Bürgerbegehren und Bürgerentscheiden unter den Initiatoren wie auch den Abstimmenden ressourcenstarke Bürger, d.h. solche mit stärkerem politischen Interesse, höherer Bildung und ausgeprägtem bürgerschaftlichen Selbstbewusstsein (political efficacy), überdurchschnittlich vertreten sind. ${ }^{5}$

Mit der weiteren Frage, ob Plebiszite ein Einfallstor zur Ausgrenzung von Minderheiten per Stimmzettel öffnen, wird zweifellos ein elementares Praxisproblem für die Belastbarkeit demokratischer Grundprinzipien formuliert. Auch in dieser Hinsicht ist der Forschungsstand differenziert und benennt folglich auch mögliche Nachteile und Risiken für Minoritäten: Bärbel Weixner zufolge, deren Studie auch Frank Decker heranzieht, verhindern eben die (von Befürwortern direkter Demokratie hierzulande ausdauernd kritisierten) Eingangsquoren für Volksinitiativen und Volksentscheide, dass sich auf der Landesebene der Bundesrepublik Anliegen durchsetzen können, die lediglich von kleinen Minderheiten getragen werden. ${ }^{6}$ Auch laut John Haskill, der US-amerikanische Studien umfassend ausgewertet hat, sollten direktdemokratische Instrumente als Vehikel für die aktive Durchsetzung von Parti-

4 Marie-Luise Schneider, Vom Nutzen von Volksabstimmungen, in: WZB-Mitteilungen 2004, H. 103, S. 28.

5 So schon Oscar W. Gabriel, Das Volk als Gesetzgeber: Bürgerbegehren und Bürgerentscheid in der Kommunalpolitik aus der Perspektive der empirischen Forschung, in: Zeitschrift für Gesetzgebung, 14. Jg. (1999), H. 4, S. 299 - 331. So lässt sich für Hamburger Volksentscheide schon vor dem öffentlich vielbeachteten Volksentscheid zur Schulreform 2010 aufzeigen, dass in Bezirken mit besserem Ausstattungs- und Sozialprofil der Bewohner, gemessen an den Indikatoren Arbeitslosenrate und Wohnfläche, die Beteiligung auch bei Volksentscheiden höher liegt (vgl. Romy Heimann, Direkte Demokratie auf Landesebene am Beispiel Hamburgs - Akteure und Ressourcen, unveröffentlichte Magisterarbeit, Halle (Saale) 2008). Vgl. neuestens Everhard Holtmann, Bürgerbegehren als Weg lokaler Demokratie?, in: Der Städtetag 2010, H. 6, S. 7 - 9.

6 Vgl. Bärbel M. Weixner, Direkte Demokratie in den Bundesländern, Opladen 2002, S. 268. 
kularinteressen (,the ability of narrow interests to get what they want through direct democracy “7) nicht überbewertet werden. Außerdem bedienen sich, so fährt Haskill fort, benachteiligte Minderheiten in den USA nachweislich nicht der plebiszitären Verfahren, um ihre Interessen gezielt geltend zu machen. ${ }^{8}$ Und in ihrer Analyse von Schweizer Volksabstimmungen von 1960 bis 2007 kommen Adrian Vatter und Deniz Danaci zu dem Ergebnis, dass Minderheiten diskriminierende Effekte direkter Demokratie in der Schweiz „mit dem gesellschaftlichen Integrationsgrad der betroffenen Minderheit und ihrer Wahrnehmung als Fremdgruppe“ variieren. Insofern trage die direkte Demokratie „durchaus das Potenzial einer Mehrheitstyrannei in sich"9.

Schon dieser ausschnitthafte Blick auf den Forschungsstand zur direkten Demokratie zeigt, dass im Falle von Vorschlägen zu einer Verfassungsreform, die wie die Direktwahl des Ministerpräsidenten das institutionalisierte Verhältnis der Gewalten im Regierungssystem Deutschlands im Kern berührt, eine präventive Folgenabschätzung der beabsichtigten Reform - soweit vor dem vorhandenen Erfahrungshintergrund möglich - unverzichtbar ist. Dies gilt für die grundsätzliche demokratiepraktische Güterabwägung einer Ausweitung volksunmittelbarer Entscheidungsrechte auf die Wahl der Ministerpräsidenten ebenso wie für die konkrete Frage, ob - und wenn ja, welche - Wirkungen in der Folge des konstitutionellen „Nebeneffekts" einer durch direkte Wahl der Regierungsspitze insgesamt beschnittenen repräsentativen Demokratie möglicherweise eintreten könnten, die die politisch-administrative Praxis demokratischen Regierens erschweren beziehungsweise den Parlamentsvorrang tendenziell unterlaufen. Anders gesagt: Welche Auswirkungen auf die innere Machtbalance, die Handlungsmuster und die Effizienz eines präsidentialistisch „,runderneuerten“ Regierungssystems der Bundesländer sind zu erwarten, wenn mittels Direktwahl des Regierungschefs „die Regierungsform auf das bestehende System der Direktdemokratie zugeschnitten "10 würde?

Diese Frage wird in Frank Deckers Beitrag weitgehend ausgespart, einmal abgesehen von den unmittelbar eintretenden und allgemein bekannten primären Folgen einer derartigen Systemtransformation. Unbestritten ist, dass mit einem direkt gewählten Ministerpräsidenten die im parlamentarischen System vorhandene Anordnung der Gewalten, mit der die Linien von Kooperation und Konflikt zwischen parlamentarischem Gesetzgeber und gouvernemental geführter Exekutive bislang markiert werden, sich zwangsläufig verändern würde: Im Zuge einer Präsidentialisierung des parlamentarischen Regierungssystems wäre die dieses System tragende politische Handlungseinheit von Regierung und Parlamentsmehrheit im Parlament voneinander entkoppelt. Absehbar wäre dann ein zusätzlicher substantieller Funktions- und Machtverlust der Landtage allein schon deshalb, weil diese ihr bisheriges Recht zur Wahl und Abwahl der Landesregierung des (künftig vom Landesvolk direkt gewählten) Regierungschefs verlören (abgesehen vielleicht von einer der Präsidentenanklage entsprechenden Ausnahmeklausel). Eine weitere direkte Konsequenz wäre außer-

7 John Haskell, Direct Democracy of Representative Government? Dispelling the Populist Myth, Boulder / Oxford 2001, S. 105.

8 „There is not a single such group - representing homosexuals, African Americans, or immigrant groups - that would take its chances with a plebiscite." Ebenda, S. 111.

9 Vgl. Adrian Vatter / Deniz Danaci, Mehrheitstyrannei durch Volksentscheide? Zum Spannungsverhältnis zwischen direkter Demokratie und Minderheitenschutz, in: PVS, 51. Jg. (2010), H. 2, S. $205-222$, S. 219.

10 Frank Decker, a.a.O. (Fn. 1), S. 576. 
dem, dass das Instrument des konstruktiven Misstrauensvotums, das bisher im Falle von sich verändernden Parlamentsmehrheiten den Regierungswechsel während der Wahlperiode ermöglicht, aus den Landesverfassungen eliminiert werden müsste. Denn das „legitimatorische Gleichauf" ${ }^{\prime 11}$ zweier jeweils direkt gewählter Staatsorgane schließt die Abwahl des einen durch das andere naturgemäß aus. Mit dem Wegfall des konstruktiven Misstrauensvotums entfielen auch jene ihm in der parlamentarischen Praxis vorausgehenden Vorwirkungen, also der Anreiz, darauf hinzuarbeiten, gegebene instabile Majoritäten durch eine alternative Regierungsmehrheit zu ersetzen. Damit gäbe es eine Möglichkeit weniger, gegenüber der Regierung den Part aktiver parlamentarischer Führerschaft zu spielen. Ferner würde die Vertrauensfrage, mittels derer schwankende Regierungsmehrheiten parlamentarisch restabilisiert oder aber, bei Scheitern der Aktion, in die vorzeitige Auflösung des Landtags münden können, systemwidrig. Mit der Abschaffung dieses Instruments verlören die landespolitischen Akteure eine Möglichkeit, auf ernstere Regierungskrisen flexibel zu reagieren. $^{12}$

Dass diese Verschiebung im Verhältnis der legislativen und exekutiven Gewalten einträte, ist unstreitig und findet auch bei Decker in einer Fußnote Erwähnung. ${ }^{13}$ Doch was folgt weiter aus der verfassungsrechtlichen Neukonfiguration der landesstaatlichen Organe? Meine These ist, dass mit hoher Wahrscheinlichkeit solche Sekundäreffekte zu erwarten wären, deren Blockaderisiko und Dysfunktionalitätspotential beträchtlich sind. Diese These wird im Folgenden für den hier diskutierten deutschen Fall anhand dreier ausgewählter Problempunkte näher ausgeführt: (1) an den Folgen des Fortfalls der Sperrklausel, (2) an den Unwägbarkeiten der neuen Gewaltentrennung in einem mit „monokratischer“ Machtbasis ausgestatteten Ministerpräsidenten-Regime und (3) an den Herausforderungen für eine zwischen getrennten Gewalten arbeitende staatliche Bürokratie. Bei letzterem Punkt sind die beiden Dimensionen der Inter-Organ-Beziehungen und der Intra-Organ-Relationen analytisch zu unterscheiden.

\section{Obsolete Sperrklauseln, stärker fragmentierte Landtage, beeinträchtigte Regierbarkeit?}

Im Gefolge der Einführung einer volksunmittelbaren Wahl des Ministerpräsidenten würde die geltende Fünf-Prozent-Sperrklausel bei Landtagswahlen verfassungsrechtlich obsolet. Das mit der Sperrklausel einhergehende Demokratiedefizit, das heißt die Verzerrung des Erfolgswerts gültig abgegebener Stimmen sowie die Einschränkung der Chancengleichheit kandidierender Parteien, gegen das funktionale Pro-Argument einer erhöhten Gewährleistung parlamentarisch basierter Regierungsfähigkeit abwägend, hat das Bundesverfassungsgericht seit den frühen 1950er Jahren in fortlaufender Rechtsprechung die Fünf-Prozent-

11 So die einprägsame Wortschöpfung des Staatsrechtlers Fabian Wittreck in seinem Thesenpapier für den Gesprächskreis Zukunft der Parteiendemokratie der Friedrich-Ebert-Stiftung am 28./29. Oktober 2010 in Berlin (unveröffentlicht).

12 Das Instrument der Vertrauensfrage ist mit Ausnahme Niedersachsens, Nordrhein-Westfalens und Baden-Württembergs in sämtlichen Landesverfassungen verankert: Art. 44 BayVerf, Art. 57 BlnVerf, Art. 87 BbgVerf, Art. 110 BremVerf, Art. 36 HmbVerf, Art. 114 HV, Art. 51 MVVerf, Art. 99 RhPfVerf, Art. 69 SächsVerf, Art. 74 LSAVerf, Art. 88 SVerf, Art. 36 SHVerf, Art. 74 ThürVerf.

13 Vgl. Frank Decker, a.a.O. (Fn. 1), S. 576, Fn. 43. 
Barriere für grundsätzlich verfassungskonform erklärt. ${ }^{14}$ Dabei wog „die Sicherung der Funktionsfähigkeit der zu wählenden Volksvertretung“"vergleichsweise schwer:

„Für Wahlen zu gesetzgebenden Körperschaften hat das Bundesverfassungsgericht mehrfach ausgeführt, die Verhältniswahl könne dazu führen, dass im Parlament viele kleine Gruppen vertreten sind und hierdurch die Bildung einer stabilen Mehrheit erschwert oder verhindert wird. [So] bestünde die Gefahr, dass die gesetzgebenden Körperschaften funktionsunfähig werden, insbesondere nicht mehr in der Lage sind, eine politisch aktionsfähige Regierung zu schaffen (vgl. BVerfGE 1, 208 [248]).“15

Aufschlussreich für das uns hier beschäftigende Thema einer Reform der Landesverfassungen ist nun, dass das BVerfG, als es im Februar 2008 die Sperrklausel im kommunalen Wahlgesetz Schleswig-Holsteins kippte, ausdrücklich mit der zuvor flächendeckend eingeführten präsidentiellen Umformung des kommunalen Entscheidungssystems argumentierte: Da der inzwischen direkt gewählte und mit materiell weitreichenden Leitungskompetenzen ausgestattete (Ober)Bürgermeister in seinem Organstatus nicht mehr unmittelbar von der Ratsmehrheit abhänge, sei dieser auf eine stabile Bürgermeistermehrheit im Gemeinderat künftig auch nicht mehr unmittelbar angewiesen:

„Die Direktwahl des Bürgermeisters - Entsprechendes gilt für den Landrat - garantiert bereits weitgehend eine funktionierende Gemeindeverwaltung unabhängig von den Mehrheitsverhältnissen in der Gemeindevertretung [...] Denn der Bürgermeister trägt in eigener Zuständigkeit die alleinige umfassende Verantwortung für die Leitung der Gemeindeverwaltung, für die sachliche und wirtschaftliche Erledigung der Aufgaben und für den Geschäftsgang der Verwaltung." 16

Umgekehrt würden nach Auffassung des BVerfG die gewählten Gemeindevertretungen auch nach dem Wegfall der kommunalen Sperrklausel in ihrer quasi-parlamentarischen Arbeit nicht funktionsunfähig:

„Auch bei einer größeren Anzahl von Fraktionen oder Einzelvertretern in der Gemeindevertretung oder im Kreistag drohen keine nachhaltigen Gefahren für die Fähigkeit der Kommunalvertretung, Beschlüsse zu fassen und Wahlen erfolgreich durchzuführen."17

Nach dem Wegfall der kommunalen Sperrklauseln in allen deutschen Flächenländern trat ein, was aus politikwissenschaftlicher Sicht zum Zeitpunkt der Karlsruher Verhandlungen zwar empirisch nicht hinreichend belegbar war (und auch heute noch näherer Untersuchung bedarf), was aber aufgrund der Kenntnisse über die Mechanismen und Modalitäten ratszentrierter Entscheidungsbildung, die in der lokalen Politikforschung in etlichen Fallstudien seit längerem vorliegen, mit hoher Wahrscheinlichkeit erwartet werden durfte: Zum einen ist im Laufe des letzten Jahrzehnts die Parteienvielfalt in den Rathäusern mess-

14 Vgl. mit Verweisen auf vorausgegangene frühere höchstrichterliche Entscheidungen das Urteil des Zweiten Senats vom 13. Februar 2008 zur Verfassungswidrigkeit der Fünf-Prozent-Hürde bei Kommunalwahlen in Schleswig-Holstein (BVerfG, 2 BvK 1/07).

15 Ebenda, Rn. 120.

16 Ebenda, Rn. 133. In Reaktion auf das BVerfG-Urteil von Februar 2008 haben die Landesverfassungsgerichte Thüringens und Nordrhein-Westfalens kommunale Sperrklauseln ebenfalls für unzulässig erklärt (vgl. Thüringer Verfassungsgerichtshof, VerfGH 22/05 vom 11. April 2008 und Verfassungsgerichtshof NRW, VerfGH 12/08 vom 16. Dezember 2008). In Rheinland-Pfalz schaffte der Landtag am 14. Mai 2008 die im Kommunalwahlgesetz bis dahin geltende 3,03-Prozent-Sperrklausel ab.

17 BVerfGE, a.a.O. (Fn. 14), S. 52. 
bar durchschnittlich größer geworden; dies ist nicht ausschließlich, aber doch auch eine Folge des nunmehr unbeschränkten Verhältniswahlrechts. ${ }^{18}$ Zum anderen erschweren diese gestiegene Fragmentierung ${ }^{19}$ der kommunalen Vertretungskörperschaften und die damit einhergehende Dynamik stärkerer Interessendiffusion und Gruppenkonkurrenz in den Gemeinderäten mutmaßlich die Konsensfindung innerhalb der Ratsvertretungen wie auch ein reibungsarmes und dauerhaftes Kooperieren zwischen Ratsmehrheit(en) und Bürgermeister. In dem Maße, wie im Rat Interessengegensätze und Verteilungskämpfe fordernder artikuliert werden, gewinnt der Parteienwettbewerb in den Rathäusern an Intensität und Schärfe. „Anzunehmen ist“, schrieben Politikwissenschaftler des Sonderforschungsbereichs 580 an der der Universität Halle im November 2007 in einer Expertise für das Bundesverfassungsgericht, „dass diese Effekte der Parteiendifferenz durch eine erhöhte Fragmentierung verstärkt werden, und zwar insbesondere in mittleren und größeren Städten, wo beide Größen, also Parlamentarisierung und Fragmentierung, besonders ausgeprägt sind. “20

Diese Einschätzung der Fragmentierungseffekte wird durch ein Gutachten, das Bochumer Verwaltungswissenschaftler ein gutes Jahr später vorgelegt haben, bekräftigt. Schon kurz nach Abschaffung der kommunalen Sperrklausel in NRW erweise sich, dass die Funktionsfähigkeit der Kommunalvertretung insbesondere in den Großstädten Nordrhein-Westfalens gefährdet sei: Die Zahl der Ratsfraktionen, Gruppierungen und Einzelbewerber habe zugenommen (auf durchschnittlich acht); eine „positive gestaltende“ Mehrheitsbildung werde erschwert, unter anderem auch deshalb, weil viele kleine Gruppierungen „aufgrund ihrer Binnenstrukturen als kaum koalitionsfähig gelten“; die Fragmentierung der Räte führe „zu einer geringeren Sitzungs- und Verwaltungseffizienz“; keineswegs könne die Verwaltung und Verwaltungsspitze „weitgehend unabhängig von den konkurrenzdemokratischen Kommunalparlamenten in NRW, funktionieren“". Das Gutachten mündet in die Empfehlung, im nordrhein-westfälischen Kommunalwahlrecht eine einheitliche gesetzliche Sperrklausel von 2,5 Prozent wieder einzuführen. ${ }^{21}$

Der Analogieschluss, dass es in der landesparlamentarischen Arena nach Wegfall der Sperrklausel bei Landtagswahlen zu vergleichbaren Effekten kommt, liegt nahe - auch und

18 In einer Sachverständigen-Stellungnahme für die mündliche Verhandlung vor dem Zweiten Senat des BVerfG am 28. November 2007 bestätigte eine Forschergruppe der Universität Halle auf der Basis eines Wahldatenspeichers für die Zeitspanne von 1994 bis 2006 einen zumindest geringen restringierenden Effekt der Sperrklausel: „Eine kommunale Sperrklausel wirkt sich auf die Fragmentierung gemeindlicher Ratsvertretungen nachweisbar, aber auf sehr niedrigem Niveau, restriktiv aus. Insgesamt stieg die Anzahl der im Rat vertretenen Gruppierungen leicht an." Everhard Holtmann / Marion Reiser / Tobias Jaeck, schriftliche Stellungnahme vom 20. November 2007 (unveröffentlicht).

19 Als Indikator für Fragmentierung, also die effektive Zahl der im Rat vertretenen Parteien, können entweder die Stimmenanteile oder die Mandatsanteile herangezogen werden. Vgl. Markku Laakso I Rein Taagepera, Effective Number of Parties: A Measure with Application to West Europe, in: Comparative Political Studies, 12. Jg. (1979), H. 1, S. 3 - 27.

20 Everhard Holtmann / Marion Reiser / Tobias Jaeck, a.a.O. (Fn. 18).

21 Vgl. Jörg Bogumil / Stephan Grohs / Lars Holtkamp, Auswirkungen der Abschaffung der kommunalen 5\%-Sperrklausel auf das kommunalpolitische Entscheidungssystem in NRW, Wissenschaftliches Gutachten erstellt im Auftrag der SPD-Fraktion im nordrhein-westfälischen Landtag, Ruhr-Universität Bochum, April 2009 (unveröffentlicht), S. 3 f; dies., Zersplitterte Kommunalparlamente oder Stärkung lokaler Demokratie? Warum die Abschaffung der kommunalen Fünfprozenthürde in Nordrhein-Westfalen ein Fehler war, in: ZParl, 41. Jg. (2010), H. 4, S. 788 803. 
gerade dann, wenn man die von Frank Decker hervorgehobene strukturelle Konvergenz der Ebenen von Land und Kommunen nicht erkennen mag. Angemerkt sei hierzu, dass zum einen der Gemeinderat de jure Teil der einen kommunalen Verwaltung ist. Zum anderen verfügt der direkt gewählte Bürgermeister als Haupt der Verwaltung, als weisungsbefugter Vorgesetzter der Beigeordneten, als stimmberechtigtes Mitglied und (in den meisten Ländern) auch Vorsitzender des Rates sowie seiner Ausschüsse über eine „präsidentielle“ Machtfülle, die ein volksunmittelbar gewählter Ministerpräsident nach entsprechender Verfassungsreform wahrscheinlich nicht in gleichem Maße bei sich würde bündeln können. Eine derartige Dominanz der politischen und administrativen Führerschaft der Exekutive auf die Ebene der Landespolitik zu übertragen, ist mit einem Demokratieverständnis, das auf Mäßigung und Ausbalancierung der Organgewalten setzt, schwer vereinbar. Damit aber stünde die zentrale verfassungsgerichtliche Argumentationsfigur, dass aufgrund der Machtfülle der direkt gewählten Spitze der Exekutive die Funktionsfähigkeit des Regierens weitgehend von dieser Seite her gesichert werden könne, für die Landesebene auf schwachen Füßen.

Dass die Landtage, wenn sie im Schatten des neuen Präsidentialismus agieren müssten, jene politischen Funktionserfordernisse, die seitens der Regierung(sspitze) nicht selbstsouverän erledigt werden, ihrerseits erfüllen könnten, erscheint vor dem Hintergrund der oben erwähnten kommunalen Erfahrungen zumindest fraglich. Zwar wäre durchaus denkbar, dass Landesparlamente, wenn sie künftig stärker fragmentiert sein sollen, einem Ministerpräsidenten, der direkt gewählt und mit der angemessenen präsidentiellen Eigenkompetenz ausgestattet ist, Spielräume für projektbezogen wechselnde Mehrheiten eröffnen. In der Folge könnte sich daraus sogar eine Tendenz zu jenem aus lateinamerikanischen Ländern bekannten „Koalitionspräsidentialismus“ ergeben, bei dem Vertrauensleute der parlamentarischen „Präsidentenmehrheit“ in die präsidentiell geführte Regierung geholt werden. $\mathrm{Ob}$ eine solche „Parlamentarisierung“ des präsidentiellen Systems stattfindet, hängt jedoch unter anderem von der Kompetenzfülle ab, die dem Haupt der Exekutive, in unserem Fall dem Ministerpräsidenten, verfassungsrechtlich zugestanden oder vorenthalten wird. ${ }^{22}$

Realistischer erscheint jedoch - zumal dann, wenn man Befunde der vergleichenden Koalitionsforschung heranzieht ${ }^{23}$ - die Annahme, dass die politischen Transaktionskosten, die im präsidentialisierten Regierungssystem des Landes sowohl seitens der Regierung als auch parlamentsintern künftig aufgewendet werden müssten, um tragfähige Mehrheitsentscheidungen herbeizuführen, deutlich steigen: Kleinparteien mit Single-Issue-Profil, die vom Fortfall der Sperrklausel profitieren, schaffen nicht nur leichter den Einzug ins Landesparlament, sondern können in stärker fragmentierten Landtagen auch in die vorteilhafte Lage von Vetospielern mit hohem Erpressungspotential rücken. Gesetzesvorbereitende Sondierungs- und Aushandlungsprozesse werden länger dauern, weil sie jetzt nicht mehr durch den Kitt koalitionärer Disziplinierung und durch das Band der parteipolitisch überformten

22 Vgl. Steffen Kailitz, Der stille Abschied von der „separation of powers“. Über die „Parlamentarisierung“ präsidentieller Demokratien, in: Sabine Kropp / Hans-Joachim Lauth (Hrsg.), Gewaltenteilung und Demokratie. Probleme der „horizontal accountability“ im interregionalen Vergleich, Baden-Baden 2007, S. 168 - 190.

23 Ein Befund der Koalitionsforschung ist, dass mit der Zahl der im Kabinett vertretenen Parteien das Risiko eines vorzeitigen Scheiterns der Regierung ansteigt. Vgl. Sabine Kropp, Koalitionsregierungen, in: Oscar W. Gabriel / dies. (Hrsg.), Die EU-Staaten im Vergleich, Wiesbaden 2008, S. 514 - 549. Generell lässt sich für Westeuropa ein Zusammenhang zwischen Stabilität der Regierung und dem Fragmentierungsgrad des Parteiensystems aufzeigen. 
Loyalität, das Regierung und Regierungsfraktionen bisher verbindet, zusammengehalten werden. Zugleich werden erzielte Vereinbarungen auch „teurer“, da jetzt öfter mehr gruppenspezifische Ansprüche zu berücksichtigen sind. Außerdem werden noch häufiger Problemlösungen der Art zu erwarten sein, die noch weniger innovativ und noch stärker StatusQuo-orientiert sind - politisches Wagniskapital wird nämlich allenfalls innerhalb stabiler Koalitionsregierungen investiert. Für das Wahlvolk schließlich wird es künftig noch schwieriger werden, klar zu identifizieren, welche der Parteien im Parlament für welche Sachentscheidung jeweils verantwortlich ist.

Und schließlich: Öffnete man die Schleusen für eine stärkere Fragmentierung des Länderparlamentarismus, so würde dies mutmaßlich nicht ohne Folgen bleiben für die nächsthöhere Ebene des Bundes. Auch hier könnten Tendenzen einer erhöhten Zersplitterung des Parteiensystems befördert werden. Ein derartiger „Kamineffekt“ ist aufgrund der vertikalen Verzahnungen im bundesdeutschen Mehrebenensystem jedenfalls nicht auszuschließen. Allein eine bereits hinreichend erkennbar gewordene Evidenz der oben beschriebenen erhöhten Störanfälligkeit eines präsidentialistisch umgeformten Regierens auf der Landesebene würde dann wohl verhindern, dass auch das parlamentarische Regierungssystem im Bund entsprechendem Reformdruck ausgesetzt würde.

Nun ließe sich einwenden, die geschlossene Gesellschaft der etablierten Parlamentsparteien werde sich dem Ansinnen, im Beipack zur Einführung der Direktwahl des Ministerpräsidenten gleich auch die Sperrklausel abzuschaffen, aus ihrem wohlverstandenen Allparteienkalkül heraus gewiss verweigern. Dafür spricht in der Tat einiges. Jedoch wäre die Bresche alsbald geschlagen. In diesem Fall würden Kleinparteien mit Sicherheit Verfassungsklage einreichen, und deren Erfolgschancen sind aufgrund der oben beschriebenen Verfassungsrechtsprechung zu kommunalen Wahlen günstig einzuschätzen.

\section{Unwägbarkeiten eines „monokratischen“ Ministerpräsidenten-Regimes}

Eine Direktwahl des Ministerpräsidenten akzentuiert das Element der Gewaltentrennung im Verhältnis zwischen Parlament und Regierung. Damit wird eine partielle Umverteilung bei den Kompetenzen beider Organe notwendig. Diese Verschiebung von Zuständigkeiten geschieht nach den Regeln eines Nullsummenspiels: Was die Exekutive gewinnt, verliert der Landtag, und umgekehrt. Während beispielsweise die Exekutive - jedenfalls unter dem Gesichtspunkt einer konsequent praktizierten „Systemreinheit“ - dem Landtag folgerichtig künftig die alleinige Gesetzesinitiative überlassen müsste, sollte der direkt gewählte Regierungschef seinerseits über jene Mindestausstattung für präsidentialisiertes Regieren verfügen können, die in der Literatur als deutlich getrennte Gewalt sowie als Übertragung klarer exekutiver Verantwortung auf eine Person beschrieben wird. ${ }^{24}$ Dieser Typus politisch-administrativer Führung wird in der Kommunalliteratur als „monokratisch“ bezeichnet. $^{25}$

24 Vgl. Thomas Poguntke / Paul Webb, The Presidentialization of Politics in Democratic Societies: A Framework for Analysis, in: dies. (Hrsg.), The Presidentialization of Politics. A Comparative Study of Modern Democracies, Oxford 2005, S. 1 - 25.

25 Vgl. Melanie Walter-Rogg / Volker Kunz / Oscar W. Gabriel, Kommunale Selbstverwaltung in Deutschland, in: Oscar W. Gabriel / Everhard Holtmann (Hrsg.), Handbuch Politisches System der Bundesrepublik Deutschland, München / Wien 2005, S. 429. 
Dem US-amerikanischen Prinzip der „Checks and Balances“ deutlicher getrennter, aber zugleich zu gemeinsamer Governance zusammengeführter Gewalten entspräche es, dass die Landesregierung ihr Hoheitsrecht über den von ihr aufgestellten Entwurf des Landeshaushalts, das sie während der laufenden Etatberatungen bis zur Verabschiedung des Gesetzeswerkes ausübt ${ }^{26}$, auch weiterhin behält. Außerdem wäre ein aufschiebendes Vetorecht des Ministerpräsidenten gegenüber Gesetzesbeschlüssen des Landtags angemessen. Die Inkompatibilität von Landtagsmandat und Regierungsamt, die heute schon durch einige Landesverfassungen (zum Beispiel Bremens) vorgeschrieben wird, würde zum Regelfall werden müssen.

In dieser Projektion treten indes etliche Probleme des Regierens zutage, die dann systemtypisch würden. Eines bezieht sich auf die Vertretung der Landesinteressen im Bund. Wenn, wovon auszugehen ist, das Stimmrecht im Bundesrat weiterhin regierungsseitig ausgeübt wird, dann gewinnt die auf dieser föderal-staatlichen Ebene bekanntlich ohnedies dominierende exekutive Führerschaft zusätzliches Gewicht. Höchstwahrscheinlich würde sich auch im Rahmen der neuen gewaltenteiligen Konstellation ein informales Zusammenwirken zwischen Regierungschef, Regierung und den diese tragenden Parlamentsfraktionen herausbilden, das schon im heutigen parlamentarischen Regierungssystem die Koalitionspraxis kennzeichnet und den Mehrheitsparteien der Landtage eine materielle Einflussnahme auf das Abstimmungsverhalten der Regierungsvertreter im Bundesrat eröffnet. Auch eine schriftliche Vereinbarung der Kooperation in Form eines Koalitionsvertrags wäre ohne weiteres vorstellbar, einschließlich der zur Wahrung der Interessen kleinerer Koalitionspartner längst üblich gewordenen Bundesratsklausel, die bei Nichteinigung zur Stimmenthaltung im Bundesrat verpflichtet. Immerhin verlöre eine solche Klausel künftig einen Gutteil ihrer bisherigen Drohwirkung: Dem Ministerpräsidenten käme bei einem Verstoß gegen die Abmachung vielleicht seine bestehende parlamentarische Mehrheit, womöglich auch der eine oder andere Minister abhanden, aber zum Sturz der Regierung käme es zwangsläufig eben nicht.

Indes: Das eben beschriebene Szenario informalen Zusammenwirkens gilt für parteipolitisch gleichgerichtete Verhältnisse in Regierungsspitze und Parlamentsmehrheit, also für den durchaus wahrscheinlichen Fall, dass auch im Ergebnis getrennter Wahlen von Ministerpräsident und Landtag die gewohnten Wählerpräferenzen und hergebrachten regionalen Stärkeverhältnisse der Parteien zunächst fortgeschrieben werden. Dies dürfte vor allem dann der Fall sein, wenn beide Organe am selben Tag gewählt werden. Sehr viel schwieriger dürfte die Koordination werden, sobald Regierungschef und Majorität im Landtag konträren Parteilagern angehören. In diesem Fall eines „divided government“ ist damit zu rechnen, dass Agenden der Landesgesetzgebung - hier sitzt das Parlament am längeren Hebel - und Abstimmungen im Bundesrat - die in die Kompetenz der Landesregierung fallen in bisher nicht gekannter Form zu einer gemeinsamen Tauschmasse zusammengeführt werden: Biete Entgegenkommen beim kommunalen Finanzausgleichsgesetz und erwarte dafür Zugeständnis beim Votum zur Erhöhung der Bafög-Sätze in der Länderkammer, und so fort. Da die Klammer einer Koalition wie auch die konsensfördernde Kraft gemeinsamer programmatischer Grundüberzeugungen fehlen, würden politische Sachfragen mehr als zu-

26 Vgl. zum Budget and Accounting Act von 1921 in den USA Klaus Stüwe, Das politische System der USA, in: ders. / Stefan Rinke (Hrsg.), Die politischen Systeme in Nord- und Lateinamerika, Bonn 2010, S. 540 - 582, S. 549, S. 557. 
vor in lager- beziehungsweise gruppenpolitisch kontroverse Verteilungsfragen umgewandelt werden.

Die neue Unübersichtlichkeit der Landespolitik, die bei nicht-identischer Parteibindung von Ministerpräsident und Landtagsmehrheit eintritt, erhöht nicht automatisch die Gestaltungschancen der Parlamentsseite. Ein etwaiges Kalkül, mit Hilfe der gesetzgebenden Gewalt die Landesregierung gleichsam einzumauern, liefe ins Leere, denn auch unter Bedingungen gegengerichteter Parlamentsmehrheiten verbliebe dem direkt gewählten Ministerpräsidenten eine beträchtliche exekutive Steuerungsmacht: Er ist Chef des Kabinetts und der Ministerialbehörden und damit verantwortlich für die laufenden Geschäfte der Verwaltung. Er kontrolliert die Umsetzung der vom Landtag verabschiedeten Gesetze, was bedeutet, dass das Tempo und das „Kleingedruckte“ der administrativen Ausführung (etwa auch Ausnahmeregelungen) bestimmt werden können. Auch das suspensive Veto des Ministerpräsidenten käme fallweise zum Einsatz. Ein konfrontativ aufgestellter Gesetzgeber ließe sich mit Bordmitteln der Exekutive auf diesem Weg faktisch ausbremsen. ${ }^{27}$

Dass das Parlament bei einem derartigen Clinch der Gewalten in der Öffentlichkeit als moralischer Gewinner der Kraftprobe erschiene, ist wenig wahrscheinlich. Eher könnte sich der Regierungschef in dem vordemokratischen, jedoch populären Vorurteil sonnen, er und mit ihm das Gemeinwohl seien zu Geiseln des „Parteienhaders“ geworden.

\section{Bürokratie in der Gewaltenklemme: Zuordnungsprobleme und personeller Aufwuchs}

Dass das Feld des Regierens durch die drei Pole von Parlament, Regierung und Verwaltung abgesteckt wird, ist eine allgemein bekannte Tatsache, auf die auch in diesem Beitrag schon mehrmals Bezug genommen wurde. Die enge informale Verschränkung zwischen fachlich zuständigen Beamten der Ministerien und den Fachpolitikern der Regierungsfraktionen, die den Prozess der Gesetzesvorbereitung wesentlich trägt, kennzeichnet auch die entsprechenden Handlungsmuster in der Landespolitik.

Auch hier käme es in der Folge einer präsidentiellen Umformung des Regierungssystems zu erheblichen Veränderungen. Der formalen Institutionenreform würde ein Wandel der informalen Beziehungen nachfolgen, der teilweise die Größenordnung von Strukturbrüchen annähme: Unter den heute gegebenen Bedingungen des parlamentarischen Regierungssystems kann die von den Parlamentsfraktionen zur Gesetzesvorbereitung konsultativ beigezogene Ministerialverwaltung ihre Fähigkeiten als „politische Bürokratie“ 28 nur deshalb vergleichsweise frei entfalten, weil die ihr gegenüber weisungsbefugte Hausspitze, also Minister und Staatssekretäre, als kooperierender Teilnehmer in fraktionsinterne Entscheidungsverläufe aktiv eingebunden ist. Diese Akteurkonstellation könnte die Einführung eines präsidentialisierten Regierungssystems nur solange überleben, wie Ministerpräsident

27 Vielleicht war es nicht zuletzt diese ernüchternde Perspektive, die die Architekten der rot-grünen Minderheitsregierung in Nordrhein-Westfalen davon abbrachte, ihre dem Vernehmen nach zeitweise gehegte Überlegung, nach der Landtagswahl von 2010 die CDU-geführte Landesregierung zunächst im Amt zu lassen und selbst auf dem Umweg über die Gesetzgebung mitzuregieren, in die Tat umzusetzen.

28 Vgl. hierzu die Typenbildung bei Robert D. Putnam, Die politischen Einstellungen der Ministerialverwaltung in Westeuropa, in: PVS, 17. Jg. (1976), H. 1, S. $23-61$. 
und Landtagsmehrheit parteipolitisch gleichgerichtet sind. Bei gegenläufigen Parteizugehörigkeiten würde die Vertrauensbasis, die sich im Innenverhältnis einer Regierung und der sie stützenden Parlamentsfraktionen aus der Verpflichtung auf gemeinsame Zielvorstellungen speist, abgelöst von einer Grunddisposition wechselseitigen kontrollierten Misstrauens, die eine konstruktive Zusammenarbeit wenn nicht unmöglich macht, so doch schwieriger gestaltet. Die unsichtbare Trennwand zwischen beiden Gewalten würde mit deren Auseinandertreten stärker wirksam. Zumal die Verwaltung selbst befände sich unversehens in einer Art Gewaltenklemme: einerseits verpflichtet zum Amtsgehorsam gegenüber der Regierung und andererseits gehalten, die Gesetzesbeschlüsse des Parlaments korrekt umzusetzen.

Entspannen ließe sich dieses Loyalitäten-Dilemma dann wohl nur durch den Aufbau einer parlamentsseitigen Gegenbürokratie. Diese müsste so ausgestaltet sein, dass ein leistungsfähiger parlamentarischer Assistenzkörper entsteht, der den Abgeordneten beziehungsweise den Fraktionen bei der Vorbereitung von Gesetzesinitiativen behilflich ist. Mit bloßer Umschichtung von Personal wäre es hierbei nicht getan, weil die klassische Ministerialverwaltung mit der Umsetzung der Landesgesetze und vor allem auch für den administrativen Landesvollzug von Bundesgesetzen und EU-Normen weiterhin ausgelastet sein wird. Ein solcher Aufwuchs des öffentlichen Dienstes würde allerdings in Zeiten, da die „Entbürokratisierung" im öffentlichen Bewusstsein nicht nur als Allheilmittel gilt, sondern wesentlich mit Personalabbau gleichgesetzt wird, schwerlich Jubelstürme hervorrufen.

\section{Präsidentialisierung stärkt nicht, sondern schwächt die Parteiendemokratie}

Durch die Einführung der Direktwahl der Ministerpräsidenten würde das Boot der Demokratie nicht gleich irreparabel leck geschlagen. Die Anpassungsflexibilität der Akteure in Politik und Administration an gewandelte Handlungslagen ist in der Bundesrepublik beträchtlich. Die Fähigkeit zum institutionellen Lernen, die beispielsweise auch den komplexen Prozess der deutschen Einigung begleitet hat, wird einerseits mit hoher Wahrscheinlichkeit auch nach einer Verfassungsreform, die das parlamentarische in ein präsidentielles Regierungssystem umformt, in den Institutionen dieses Systems abrufbar bleiben.

Andererseits gewönne die Praxis verhandelnder Politik eine neue Qualität, zumal unter Bedingungen parteipolitisch gegenläufiger Konstellationen in Landtag und Landesregierung. Zu erwarten wäre ein Modus kleinteiligen Regierens, da die der Landespolitik zu eigenständiger Regelung überlassenen Politikfelder häufiger auf aushandelbare, das heißt in Inhalt und Konfliktträchtigkeit eingrenzbare Sachfragen herunter gebrochen werden dürften. Die Frage stellt sich, ob eine solche Form sequentieller, das heißt von einer Verhandlungshürde zur nächsten manövrierender, kleinteiliger Politik eine bessere Alternative zur jetzigen Praxis ist.

Der demokratische Parteienstaat beziehungsweise die Parteiendemokratie wäre schließlich ein Verlierer des Systemwechsels. Präsidentielle Eigengewalt, wie sie direkt gewählten und monokratisch ermächtigten Ministerpräsidenten eignet, kann das unterschwellige Grundgefühl, es ginge „un“" besser ohne Parteipolitiker und Parteien, sehr wohl stärken, zumal dann, wenn der Regierung eine „unbotmäßige“ Parlamentsmehrheit gegenübersteht. Gegen die Versuchung, sich in politischen Kraftproben mit dem Landtag öffentlichkeitswirksam als Hüter des Gemeinwohls zu stilisieren, dürfte keine sich selbst und plebiszitärer Akklamation überlassene exekutive Führerschaft gefeit sein. Angebliche Überparteilichkeit 
der Exekutive würde dann nicht mehr nur eine „Lebenslüge des Obrigkeitsstaates“ (Gustav Radbruch), sondern auch des präsidentialisierten deutschen Bundesstaates sein. ${ }^{29}$

Die Perspektive der Transformation der parlamentarischen zur präsidentiellen Demokratie erhält nicht schon dadurch höhere Weihen, dass neben der Mehrheit der Bevölkerung auch eine signifikante Minderheit der deutschen Landesparlamentarier eine Direktwahl des Ministerpräsidenten gutheißt. Der zweiten deutschen Abgeordnetenbefragung zufolge unterstützten jeweils mehr als 20 Prozent der Landtagsabgeordneten von CDU/CSU, SPD und Bündnisgrünen einen solchen Vorschlag; in den Reihen der Linken waren es mehr als zwei Drittel. ${ }^{30}$ Eine aktuelle Bevölkerungsumfrage im Jahr 2010 ergab, dass sich rund 83 Prozent der Bürger Sachsen-Anhalts und immerhin 27 von insgesamt 97 Abgeordneten des Magdeburger Landtages für die Volkswahl des Ministerpräsidenten aussprechen. ${ }^{31}$

Auch hier stellt sich die Frage, inwieweit einer solchen Präferenz tatsächlich eine sorgsame Abwägung der erwartbaren Konsequenzen einer derartigen Verfassungsreform unterliegt. Die Politikwissenschaft zumindest sollte geltend machen, dass es unerwünschte Folgen haben kann, wenn die Instrumente der Volkswahl und Volksgesetzgebung zum Standbein und Institutionen, die zum Kernbereich des Regierungssystems gehören, zum Spielbein von Verfassungsänderungsvorschlägen gemacht werden.

29 Derartige Ausprägungen eines präsidentiellen Politikstils sind in der Vergleichenden Systemforschung herausgearbeitet worden von Juan Linz, The Perils of Presidentialism, in: Journal of Democracy, 1. Jg. (1990), H. 1, $51-69$.

30 Vgl. Heinrich Best / Michael Edinger / Karl Schmitt / Lars Vogel, Zweite deutsche Abgeordnetenbefragung 2007 (ausgewählte Ergebnisse), Sonderforschungsbereich 580 Jena/Halle, Teilprojekt A3: Delegationseliten nach dem Systemumbruch, Jena 2007, http://www.SFB580.ProjektA3.de.vu (Abruf am 17. Dezember 2010).

31 Vgl. Everhard Holtmann, 20 Jahre parlamentarische Demokratie. Eine Analyse für Sachsen-Anhalt, in: Zwischenruf 3/2010. Das Magazin des Landtages von Sachsen-Anhalt, S. 15. 